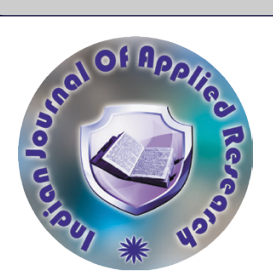

Microbiology

\title{
DEMOGRAPHIC AND EPIDEMIOLOGICAL CHARACTERISTICS OF HEPATITIS B INFECTED PATIENTS IN A TERTIARY CARE HOSPITAL
}

Bhuvaneshwar G A

Second Year MBBS Saveetha Medical College And Hospital, Thandalam, Chennai602105 .

ABSTRACT Introduction: Hepatitis B virus infection is one among the principle causes of liver diseases and death, which has limited data on demographic and epidemiological characteristics. This study aims to determine the above characters in patients diagnosed with HBV, enrolled at saveetha medical college and hospital.

Methods: Medical records of patients aged $>18$ yrs and diagnosed with HBV infection from June 2018 to December 2018 were analysed for this study.

Result: Among 79 patients taken for study, the male and female ratio is 55:24; mean age limit of HBV cases are between 40-48 years. Few cases were found to be co infected with $\mathrm{HCV}$ and 2-3 cases were medicated workers and 21 cases received tenofovir monotherapy.

Conclusion: This study reviewed the epidemiological and demographic characteristics of HBV infected patients and revealed their role in HBV. New measures has to be implemented for curative and preventive measures to create awareness among patients.

Aim : To determine the demographic and epidemiological characteristics of Hepatitis B infected patients in a tertiary care hospital

\section{KEYWORDS : Endemic ; Genotype ; Preventive ; Sterilization ;Transmission}

\section{INTRODUCTION:}

Hepatitis B Virus (HBV) is one of the major global public health problems. HBV infection is the tenth leading cause of death and HBV related Hepatocellular Carcinoma (HCC) is the $5^{\text {th }}$ most frequent carcinoma worldwide. About 30 percent of world's population has serological evidence of current or past infection with HBV. Of these an estimated 350 million are chronically infected with HBV and approximately 1 million persons die annually from HBV related chronic liver diseases including severe complications such as Liver Cirrhosis (LC) and HCC.HBV is distributed worldwide, but its prevalence varies significantly between different populations of the world.

\section{Area of high prevalence (HbsAg Carriage $>8 \%$ ) \\ Sub-Saharan Africa}

East Asian and Alaskan Population

\author{
Area of intermediate prevalence (HbsAg Carriage > 2- $7 \%$ ) \\ Southern parts of eastern and central Europe \\ Amazon basin \\ Middle-east and Indian sub-continent
}

\section{Low HBV Endemic (HBSAG Carriage $<2 \%$ )}

Western and Northern Europe

Northern America and Australia

In India $\mathrm{HbsAg}$ prevalence among general population ranges from $2 \%$ to $8 \%$, placing India in intermediate $\mathrm{HbsAg}$ endemic zone and the number of HBV carriers is estimated to 50 to 60 million, forming the second largest global pool of chronic HBV infections. India is a vast country, comprised of multiracial communities with wide variations in ethnicity and cultural patterns, which is attributable to its geographical location, gene influx due to invasions and anthropological migrations in the past. Moreover, recent increase in trade, trafficking and use of illicit drugs has also considerably influenced the epidemiology of HBV specifically in the eastern and north-eastern parts of India. However data on the molecular epidemiology of HBV in India is scanty. Interestingly in addition to genotypes $\mathrm{A}$ and $\mathrm{D}$, genotype $\mathrm{C}$ having high nucleotide. Similarity with south-east Asian sub genotype strain, have been detected exclusively from eastern Indian HBV carriers suggesting a recent introduction. This article is focussed on to determine demographic and epidemiological characteristics of patients diagnosed with HBV and follow up at Saveetha Medical College Hospital.

\section{STUDY DESIGNAND METHODS:}

This was a retrospective, descriptive study conducted by Microbiology Department at Saveetha Medical College Hospital, Tandalam, Chennai from June 2018 to December 2018. About 5500 medical records of both outpatients and inpatients above 18 years old were reviewed for this study at Saveetha Medical College. Among these patients about 79 patients were confirmed with HBV positive infection
$\left(\mathrm{HBsAg}^{+}, \mathrm{HBeAg}^{+}\right.$, anti $\mathrm{HBcIgM}^{+}$or $\mathrm{HBV}-\mathrm{DNA}^{+}$) according to the clinical protocol.

\section{MATERIALS REQUIRED:}

Mainly Hepatitis diagnostic Serology is performed by enzyme linked immunosorbent assay for (ELISA) HBV.Hepatitis B surface antigen ( $\mathrm{HbsAg}$ ) is most frequently used to screen for the presence of this infection.However early in infection this antigen maybe present or it may be undetectable later in the infection as it is cleared by the host. During the window period IgM antibodies specific to the hepatitis B Core antigen (anti $\mathrm{HbcIgM}$ ) maybe the only serological evidence of the disease.So most of the hepatitis B diagnostic panels contain HBsAg and total anti HBC (both IgM and IgG). In some cases higher rates of viral replication and enhanced infectivity was detected by the presence of another antigen called (HbeAg) Hepatitis B e antigen.Individuals who remain $\mathrm{HBsAg}$ positive for atleast six months are considered to be the hepatitis B carriers. These chronic cases of hepatitis B are reflected by elevated serum alanine aminotransferase (ALT) levels are inflammation of liver.PCR test have been developed to detect and measure the amount of HBV DNA called as viral load.We reviewed all the assays done for confirmation of hepatitis $\mathrm{B}$ from results.Blood samples were collected from patients and sent for above test for confirmation.

\section{DATACOLLECTION:}

\section{Demographical and Epidemiological Details}

The basic information collected from the records consisted of demographic variables like sex, marital status, residence, institutionalization and educational status. Risk factors were also collected which are associated with HBV infections like Community associated : Tattoo and body piercing, dentistry treatment, family conduct and sexual transmission from husband. Hospital associated risk factors like Blood exposure and past surgery were also collected including the behavioural factors like Intranasal drug use, injectable drug use, more than one sex partners and heavy alcohol use. All the above data were collected from patients and considered for the survey.

\section{RESULTS AND DISCUSSION:}

From the demographic details collected from HBV Positive patients, from Saveetha Medical College Hospital, during the period of June 2018 to December 2018 the following results were obtained finally.About 79 patients with proven, HBV positive infection cases were enrolled in the study, and the results are calculated according demographically.

\section{Gender Group}

Totally 79 patients were admitted in the hospital with the HBV infection. Among them about 19 were female with a percentage of $24 \%$ and about 60 of them were male with percentage of $76 \%$. The male and female ratio according to the data collected is $76: 24$. This implies that the male were more affected than the female.

\begin{tabular}{|c|c|c|c|}
\hline & Submitted : 14 $4^{\text {th }}$ June, 2019 & Accepted : $29^{\text {th }}$ September, 2019 & Publication : 01 ${ }^{\text {st }}$ December, 2019 \\
\hline 6 & \multicolumn{3}{|c|}{ INDIAN JOURNAL OF APPLIED RESEARCH } \\
\hline
\end{tabular}




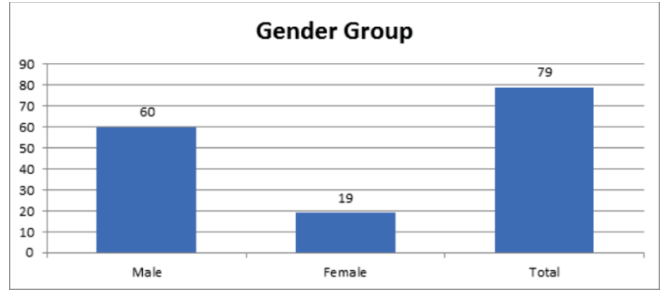

Age Group

Along with the gender, the age group of the affected was also simultaneously collected. It revealed that about only 19 patients were below the 35 years of age. And 16 patients were between $35-44$ years of age ; 20 patients were between 45 - 54 years of age $; 24$ patients were between the age of $55-75$ years of age. Thus from data, the mean age ratio was between $45-48$ years of age. The occurrence of the infection reduced with increase in age.

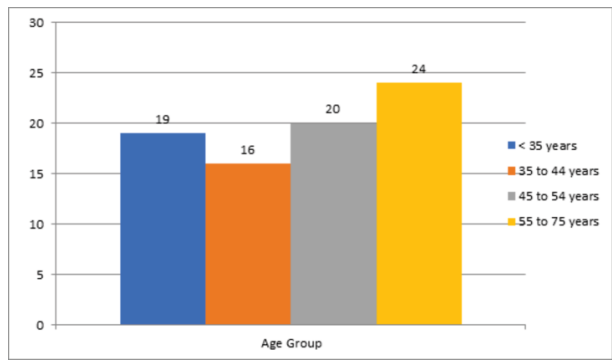

\section{Other Demographic Details}

Along with the age and gender details other demographic details were also specifically observed. About 70 patients from the collected data were married and 69 of them were living in the urban areas. About 15 patients were educated up to the college level and 30 of them were illiterate. Among these about 55 patients were occupied with some jobs while 15 of them were without any steady job. 3 of them were found to be health care workers working in hospitals.

\section{Regarding Risk factors}

Concerning the risk factors associated with HBV infection, Tattoos, ear and nose piercing and dentistry treatment was the most prevalent community associated risk factors reported by $32(42.3 \%)$ patients. Past surgery was the most prevalent Hospital associated risk factors found in $36(48 \%)$ patients and $>=3$ sexual partners was the most prevalent Behaviour associated risk factors in $27(36 \%)$ patients. HIV \& HCV co-infection was present in $17(22.77 \%)$ patients. $9(12 \%)$ were co-infected with HIV and $5(6.7 \%)$ were co-infected with HCV and $3(4 \%)$ were co-infected with both HIV and HCV. Aged people are found to be co-infected with Pulmonary Tuberculosis, CAD and chronic diseases.

Table 2. Characteristics of patients receiving Hepatitis B virus antiviral therapy

\begin{tabular}{|c|c|c|}
\hline Patients (79) & No (58) & Yes (21) \\
\hline \multicolumn{3}{|c|}{ 1. Age Category } \\
\hline$<35$ & 25 & 3 \\
\hline $35-44$ & 11 & 9 \\
\hline $45-54$ & 13 & 5 \\
\hline $55-75$ & 10 & \\
\hline \multicolumn{3}{|l|}{ 2. Sex } \\
\hline Male & 39 & 16 \\
\hline Female & 19 & 5 \\
\hline \multicolumn{3}{|c|}{ 3. ALT Status } \\
\hline Normal & 34 & 18 \\
\hline Increased & 24 & 3 \\
\hline \multicolumn{3}{|l|}{ 4. HbeAg } \\
\hline Positive & 13 & 5 \\
\hline Negative & 17 & 8 \\
\hline Not Detected & 28 & 8 \\
\hline \multicolumn{3}{|c|}{ 5. Clinical Form } \\
\hline Chronic & 40 & 19 \\
\hline Acute & 11 & 2 \\
\hline Pulminant & 2 & 1 \\
\hline
\end{tabular}

This study presents hospital based populative observations regarding epidemic demographic and risk factors for transmission of hepatitis B.
Our study analysed data from over a period of time and the patients included based on diagnosis did not represent the entire burden of HBV infection in Tamil Nadu, India.

The peak of infection occurred in individuals between $40-48$ years old and declined thereafter, $12 \%$ were co-infected with HIV, $6.7 \%$ were with HCV and $4 \%$ were with both HIV and HCV. Tenofovir Mono Therapy was the most prescribed medication. Approximately after 2 years of antiviral treatment, the HBV DNA viral load was undetectable in $92.3 \%$ patients and lower levels of ALT were found in these patients. There Is a countrywide under estimation of true number of individuals infected with HBV mostly because the highest risk population are underrepresented in surveillance. This is a significant percentage of chronically infected individuals remain undiagnosed. This situation is shared worldwide and highlights the strong likelihood that chronic Hepatitis B remains an under-recognised disease.

In India, healthcare workers are at increased risk of exposure to Hepatitis B and C. Immunisation against Hepatitis B is recommended in healthcare workers, but there is ignorance and lack of awareness regarding this. A recent study from south India concluded that knowledge and practice of universal precaution among paramedics was unsatisfactory. The risk of infection after needle stick injury is 23 (62\%) for HBV. Another study showed 29 out of 72 microbiology lab workers were susceptible for HBV infection. We found having a tattoo, ear \& nose piercing and dentistry treatment were significantly associated with HbsAg Cero positivity. Reuse of needle without proper sterilization is the main cause in the lab. Male to male sex was not reported by any other case. Acupuncture is not common here and was not reported by anybody. Haemodialysis is a risk factor for HBS As but no case was reported. None of the subjects reported history of organ transplantation. There are many potential limitations to our study. Lack of awareness regarding the disease in general population and population based nature of study has limitation. Second potential recall bias regarding the risk factors like unsafe injections. Third we excluded children less than 5 years because of technical difficulty of blood collection for test. Occult and cured infections could not be diagnosed.

\section{CONCLUSION:}

In conclusion, the result of this thesis sheds light on many important aspects of demographic and epidemiological aspects of HBV infection. These are very much important for identifying the population at risk of acquiring HBV and developing chronic liver diseases and also pose a risk of transmission through different modes. This information are essential for detecting the risk factors associated with $\mathrm{HBV}$ infection to formulate necessary preventive measures to lessen the burden of new infection and spread. As the HBV and HBC remain undiagnosed diseases, awareness should be created regarding the infections to the public who are at risk like Healthcare workers and Microbiology Lab workers etc. There is urgent need to form new policies and strategies to implement this. Educating common people regarding mode of transmission of Hepatitis B \& C will help reduce infection.

\section{REFERENCES:}

1. World Health Organization (WHO) Media Centre. Hepatitis B. 2015. (Accessed 2015 March).

2. Tandon BN, Acharya SK, Tandon A. Epidemiology of hepatitis B virus infection in India. Gut. 1996;38(Suppl2):S56-9.

3. Joint United Nations Programme on HIV/AIDS (UNAIDS). Countries: Brazil. 2014. (Accessed 2015 March).

4. Acharya SK, Madan K, Dattagupta S, Panda SK. Viral hepatitis. India Natl Med J India. 2006;19(4):203-17.

5. Jha AK, Chadha S, Bhalla P, Saini S. Hepatitis B Infection in Microbiology Laboratory Workers: Prevalence, Vaccination, and Immunity Status. Hepat Res Treat. 2012;2012:520362.

6. Singh J, Bhatia R, Patnaik SK, Khare S, Bora D, Jain DC, Sokhey J. Community studies on hepatitis B in Rajahmundry town of Andhra Pradesh, India, 1997-8: unnecessary therapeutic injections are a major risk factor. Epidemiol Infec. 2000;125(2):367-75.

7. Cohen C, Evans AA, London WT, Block J, Conti M, Block T. Underestimation of chronic hepatitis B virus infection in the United States of America. J Viral Hepat 2008; 15:12-13.

8. Clinical, demographic, and epidemiologic characteristics of hepatitis B virus-infected patients at a tertiary public hospital in Presidente Prudente, State of São Paulo, Brazil ;

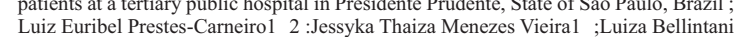
Luiz Euribe
Isaac 1

9. Luo Z, Xie Y, Deng M, Zhou X, Ruan B. Prevalence of hepatitis B in the southeast of China: a population-based study with a large sample size. Eur J Gastroenterol Hepatol 2011;23:695-700. 